\title{
Leisure-time physical activity is a significant predictor of stroke and total mortality in Japanese patients with type 2 diabetes: analysis from the Japan Diabetes Complications Study (JDCS)
}

\author{
H. Sone $\cdot$ S. Tanaka $\cdot$ S. Tanaka $\cdot$ S. Suzuki $\cdot$ H. Seino $\cdot$ \\ O. Hanyu • A. Sato $\cdot$ T. Toyonaga $\cdot$ K. Okita \\ S. Ishibashi • S. Kodama • Y. Akanuma • N. Yamada • \\ on behalf of the Japan Diabetes Complications Study Group
}

Received: 29 April 2012 / Accepted: 29 November 2012 /Published online: 27 February 2013

(C) Springer-Verlag Berlin Heidelberg 2013

\begin{abstract}
Aims/hypothesis Our aim was to clarify the association between leisure-time physical activity (LTPA) and cardiovascular events and total mortality in a nationwide cohort of Japanese diabetic patients.
\end{abstract}

A complete list of members of the JDCS group can be found in the electronic supplementary material (ESM).

Electronic supplementary material The online version of this article (doi:10.1007/s00125-012-2810-z) contains peer-reviewed but unedited supplementary material, which is available to authorised users.

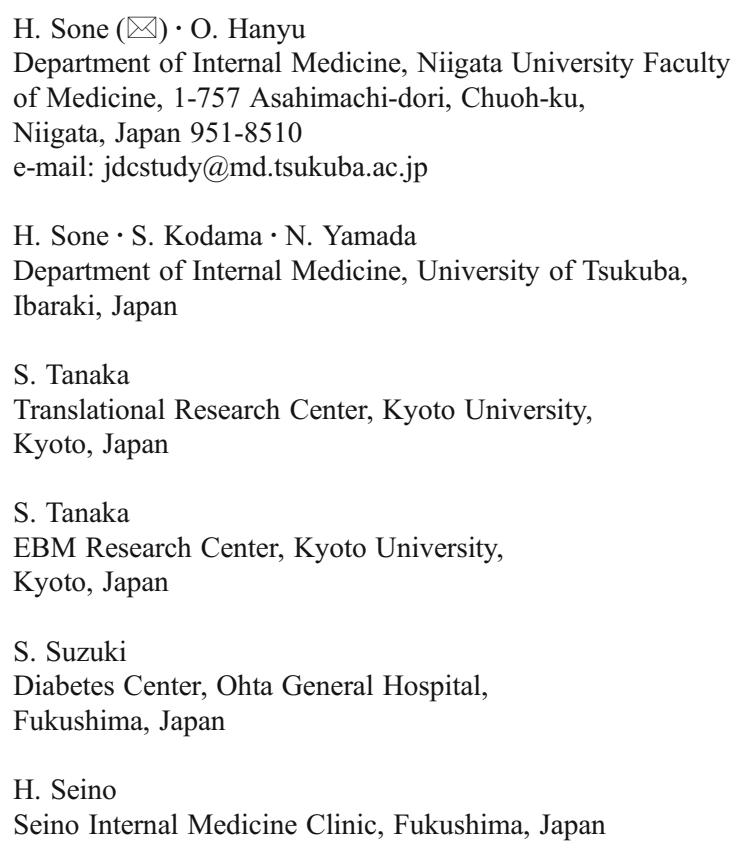

Methods Eligible patients (1,702) with type 2 diabetes (mean age, 58.5 years; $47 \%$ women) from 59 institutes were followed for a median of 8.05 years. A comprehensive lifestyle survey including LTPA and occupation was performed using standardised questionnaires. Outcome was
A. Sato

Diabetes Center, Tokyo Women's Medical University,

Tokyo, Japan

\section{T. Toyonaga}

Department of Diabetes and Endocrinology,

National Hospital Organization Kumamoto Medical Center,

Kumamoto, Japan

K. Okita

Department of Metabolic Medicine,

Osaka University, Osaka, Japan

S. Ishibashi

Department of Endocrinology and Metabolism,

Jichi Medical College, Tochigi, Japan

Y. Akanuma

The Institute for Adult Diseases Asahi Life Foundation,

Tokyo, Japan 
occurrence of coronary heart disease (CHD), stroke and total mortality. The adjusted HR and $95 \%$ CI were calculated by Cox regression analysis.

Results A significant reduction in HR in patients in the top $(\geq 15.4$ metabolic equivalents [MET] $\mathrm{h} /$ week) vs the bottom tertile ( $\leq 3.7 \mathrm{MET} \mathrm{h} /$ week) of LTPA, adjusted by age, sex and diabetes duration, was observed in stroke (HR $0.55,95 \% \mathrm{CI}$ $0.32,0.94$ ) and total mortality (HR $0.49,95 \%$ CI $0.26,0.91$ ) but not in CHD (HR 0.77, 95\% CI 0.48, 1.25). The HR for stroke became borderline significant or nonsignificant after adjustment for lifestyle or clinical variables including diet or serum lipids. The significantly reduced total mortality by LTPA was independent of these variables and seemed not to be, at least mainly, attributed to reduced cardiovascular disease.

Conclusions/interpretation In Japanese persons with type 2 diabetes, LTPA of 15.4 MET h/week or more was associated with a significantly lower risk of stroke partly through ameliorating combinations of cardiovascular risk factors. It was also associated with significantly reduced total mortality but independently of cardiovascular risk factors or events. These findings, implying differences from Western diabetic populations, should be considered in the clinical management of East Asians with diabetes.

Keywords Asian - Cardiovascular disease - Cohort study . Ethnic difference $\cdot$ Exercise $\cdot$ Macrovascular complications · Physical activity

$\begin{array}{ll}\text { Abbreviations } \\ \text { IGT } & \text { Impaired glucose tolerance } \\ \text { JDCS } & \text { Japan Diabetes Complications Study } \\ \text { JDS } & \text { Japan Diabetes Society } \\ \text { LTPA } & \text { Leisure-time physical activity } \\ \text { MET } & \text { Metabolic equivalents }\end{array}$

\section{Introduction}

Type 2 diabetes is a significant cause of premature mortality and morbidity especially related to cardiovascular disease. Regular exercise has been recommended to prevent these diabetic complications through ameliorating control of several variables related to diabetes [1-3]. Many but not all [4-6] cohort studies have shown that physical activity is prospectively associated with reduced total [7-18] and cardiovascular $[7-13,19]$ mortality. It has also been demonstrated, albeit in a few studies $[4,18,20,21]$, that cardiovascular events (i.e. coronary heart disease [CHD] and stroke) were reduced according to increased physical activity in individuals with diabetes. However, only two of those studies $[4,18]$ analysed cardiovascular events and mortality simultaneously and produced conflicting results on mortality. In addition, only three of those studies $[4,20,21]$ determined CHD and stroke separately and their results for stroke were conflicting. Therefore, it is still unclear how physical activity exerts its effect on reducing mortality in patients with diabetes and whether macrovascular complications of diabetes are reduced in accordance with increased physical activity.

Most previous studies evaluated physical activity only as non-numerical or bivariate variables, such as a self-reported response of whether exercise was done regularly [5-9, $12-14,16,20]$ or only with regard to walking $[11,19]$. Data are not abundant on dose-effect relationships regarding quantity of physical activity, especially determined in units of metabolic equivalents (MET) $\mathrm{h}[15,18,21]$. These units are universally used for quantification of physical activity and are useful for determining cut-offs in required amounts of exercise for risk reduction and exchange of values for different types of exercise and complications and mortality in patients with diabetes.

While different approaches to exercise are needed depending on ethnicity because of different responses to exercise among ethnic groups [3], all of these previous cohort studies were carried out in Western diabetic populations with only a few exceptions [4, 7]. However, it is uncertain whether results from Western diabetic patients can be extrapolated to East Asian populations with diabetes. In this analysis of data from a long-term follow-up of Japanese patients with type 2 diabetes, we analysed the association between leisure-time physical activity (LTPA), which accounts for an important part of exercise therapy for diabetic persons, and risk for CHD, stroke and total mortality.

\section{Methods}

Recruitment of patients The present analysis was conducted as part of the Japan Diabetes Complications Study (JDCS), a multicentre prospective study of the incidence of and risk factors for complications among 2,033 Japanese patients with type 2 diabetes aged $40-70$ years with $\mathrm{HbA}_{1 \mathrm{c}}$ (Japan Diabetes Society [JDS]) levels $\geq 6.5 \%$ ( $51 \mathrm{mmol} / \mathrm{mol})$ who were registered from January 1995 to March 1996 from outpatient clinics in 59 university and general hospitals nationwide that specialise in diabetes care. Of those 2,033 individuals, 940 men (mean age \pm SD $57.8 \pm 7.1$ years) and 831 women $(58.7 \pm 6.8$ years $)$ were selected for the analysis of macrovascular complications after consideration of the exclusion criteria pre-specified in the study protocol [22]. Patients were excluded if they had impaired glucose tolerance (IGT), a history of angina pectoris, myocardial infarction, stroke, peripheral artery disease, familial hypercholesterolaemia, type III hyperlipidaemia (diagnosed by a broad beta band on electrophoresis) or nephrotic syndrome (urine protein $>3.5 \mathrm{~g}$ per day and serum total protein $<60 \mathrm{~g} / \mathrm{l}$ ) and serum creatinine levels greater than $120 \mu \mathrm{mol} / \mathrm{l}(1.3 \mathrm{mg} /$ dl). In the 8-year planned observation period, the median 
follow-up for the 1,771 patients was 7.86 years (final follow-up rate was $75 \% ; 1,332 / 1,771$ patients). For this analysis of physical activity, data on the 1,702 patients (901 men, age $58.2 \pm 7.0$ years; 801 women, age $58.9 \pm 6.8$ years) who responded to the baseline physical activity survey were used. There was no notable difference in baseline characteristics between responders and non-responders. We analysed follow-up data until March 2003.

Diabetes mellitus and IGT were diagnosed according to the 'Report of the Committee of the Japan Diabetes Society on the Classification and Diagnostic Criteria of Diabetes Mellitus', which is almost identical in terms of thresholds for glucose levels to those of the WHO. The study protocol, which is in accordance with the Declaration of Helsinki and the Ethical Guidelines for Clinical/Epidemiological Studies of the Japanese Ministry of Health Labor and Welfare, received ethics approval from the institutional review boards of all participating institutes. All enrolled patients provided written informed consent.

Assessment of LTPA LTPA was assessed at baseline by a self-administered questionnaire, which was almost identical to that used and validated in the Health Professionals' Follow-up Study [18]. The patients were asked the average frequency (times/week) and duration (min/time) of normal walking, brisk walking, jogging, golf, tennis, swimming, aerobics dancing, cycling and other miscellaneous exercise (specified by each patient). The duration engaged in each activity in min/time was multiplied by that activity's typical energy expenditure, expressed in MET, based on the newest compendium of Ainsworth [23]; then overall activity was summed to yield a MET $\mathrm{h}$ score per week. The energy expended by sitting quietly, $1 \mathrm{MET}$, is equivalent to $3.5 \mathrm{ml}$ oxygen uptake (kg body weight) ${ }^{-1} \min ^{-1}$ or $4.184 \mathrm{~kJ}$ (1 kcal) (kg body weight $)^{-1} \mathrm{~h}^{-1}$.

Other lifestyle variables A baseline dietary survey, which was validated and is widely used in Japan [24], was undertaken. This consisted of food records and a food frequency questionnaire, which included questions on alcohol consumption. Information on cigarette smoking was collected using a self-administered questionnaire. Smoking status was classified into one of three categories: current smokers, ex-smokers and never smokers [25]. Occupation was surveyed by a selfadministered questionnaire based on the Japan Standard Classification of Occupations [26], which was also used in the National Health and Nutritional Examination Survey in Japan. Occupations were: (1) professional or skilled workers and technicians; (2) administrative or managerial; (3) office or clerical; (4) sales; (5) service; (6) armed force and police; (7) agricultural, forestry and fishery; (8) transport, trades and storage; (9) labourers in manufacturing, mining and construction and (10) no work or housewife. Occupations in categories
1, 2, 3 and 10 were classified as sedentary and the remainder were defined as physically active.

Clinical and laboratory measurements Patients were assessed yearly after the baseline evaluation. Mean values for at least two measurements each year were obtained for $\mathrm{HbA}_{1 \mathrm{c}}$, fasting plasma glucose and fasting serum lipids. $\mathrm{HbA}_{1 \mathrm{c}}$ assays were performed according to procedures outlined by the Laboratory Test Committee of the JDS, which is known to be converted by the formula $\mathrm{HbA}_{1 \mathrm{c}}$ (JDS) $(\%)=$ $0.98 \times \mathrm{HbA}_{1 \mathrm{c}}$ (National Glycohaemoglobin Standardisation Program; NGSP) $(\%)+0.25 \%$. All other laboratory tests were done at each participating institute. Serum LDL-cholesterol was calculated using Friedewald's equation, except where triacylglycerols exceeded $4.52 \mathrm{mmol} / 1$ (400 mg/dl), in which case LDL-cholesterol data were treated as 'missing'. This was applicable to 19 participants. All other measurements, including those for body weight, blood pressure and a 12-lead ECG, were performed at least once yearly.

Outcome measures A fatal or first non-fatal manifestation of CHD (angina pectoris or myocardial infarction) was diagnosed according to criteria defined by the Multinational Monitoring of Trends and Determinants in Cardiovascular Disease (WHO/MONICA) project. A patient with a first percutaneous coronary intervention or coronary artery bypass graft was also counted as having a CHD event. Information regarding primary outcome and other clinical variables for each individual was collected through an annual report that included detailed findings at the time of the event from each participating diabetologist who was providing care to those patients. Adjudication of endpoints was by central committees comprised of experts in diabetology as well as cardiology who were masked to risk factor status, including information on LTPA, and was based on additional data such as a detailed history, sequential changes in ECG and serum cardiac biomarkers and results of coronary angiography. Information regarding vital status and causes of death was also obtained through an annual report form and causes of death were classified based on the ninth revision of the International Classification of Diseases (ICD-9) Clinical Modification codes (www.icd9data.com/2007/ Volume1/240-279/250-259/250/default.htm) for cardiovascular disease (diagnosis codes 390-452), cancer (diagnosis codes 140-208) and other miscellaneous causes.

\section{Statistical analysis}

All statistical analyses and data management were conducted at a central data centre. Patient characteristics were described as mean $\pm \mathrm{SD}$, median and interquartile range or percentage. HRs of the incidence of each outcome for higher tertiles of LTPA compared with the lowest tertile of LTPA 
were estimated by Cox regression and also by competing risk regression, which accounts for the influence of noncardiovascular mortality when analysing associations between LTPA and CHD or stroke. These models included as confounders age, sex, diabetes duration, smoking, energy/ethanol intake, occupation (physically active/sedentary), BMI, systolic blood pressure and levels of $\mathrm{HbA}_{1 \mathrm{c}}$, LDL-cholesterol, HDL-cholesterol and triacylglycerols. The $p$ value for trend was calculated using the same regression models except that the tertile was treated as a linear term. Survival curves for each outcome according to tertiles were estimated by the KaplanMeier method. All $p$ values are two-sided and the significance level is 0.05 . All statistical analyses were conducted using SAS ver. 9.2 (SAS Institute, Cary, NC, USA).

\section{Results}

During the median follow-up period of 8.05 years, the crude incidence rates per 1,000 patient-years of CHD, stroke and death were 9.56 (95\% CI 7.95, 11.48; 114 events, 11,928 person-years), 7.40 (95\% CI 6.01, 9.11; 89 events, 12,022 person-years) and 5.60 (95\% CI 4.42, 7.09; 69 events, 12,314 person-years), respectively. The 8 -year follow-up rate was $77 \%$. Regarding causes of death, 36 deaths $(52.2 \%)$ were from cancer, 16 (23.2\%) from cardiovascular disease and sudden death, $12(17.4 \%)$ due to other known causes and $5(7.2 \%)$ due to undetermined causes. Table 1 summarises the baseline clinical characteristics of participants and the incidence of CHD, stroke and mortality according to tertiles of LTPA. The mean LTPA level of the individuals in the bottom tertile was less than $1 \mathrm{MET} \mathrm{h} /$ week and that for the middle tertile was nearly $10 \mathrm{MET} \mathrm{h} /$ week. Individuals in the top tertile had LTPA levels approximately four times higher than those in the middle tertile on average. Among these groups, the difference in age was only marginal, and there were no significant trends in sex, blood pressure, LDL-cholesterol, medication or energy intake. Significant negative trends across all groups in the degree of obesity and glycaemia and borderline significant trends in triacylglycerol levels were observed whereas there was a significant positive trend in the HDL-cholesterol level. Compared with individuals with a lower level of LTPA, those with higher LTPA included a significantly higher proportion of individuals with sedentary occupations, moderate ethanol intake and a lower smoking rate.

Table 2 shows HRs for CHD, stroke and total mortality according to tertiles of LTPA determined by Cox multivariate models adjusted as follows: by age, sex and diabetes duration (Model 1); Model 1 plus lifestyle factors (i.e. smoking, occupation and intakes of alcohol, energy, saturated fatty acids and dietary fibre [27]) (Model 2); Model 2 plus clinical variables (i.e. BMI, systolic blood pressure, $\mathrm{HbA}_{1 \mathrm{c}}$ and serum lipids)
(Model 3); and Model 3 plus medications (agents for glycaemia, hypertension or dyslipidaemia) (Model 4). These adjustments were made to clarify whether lifestyle factors other than LTPA confounded the results or whether exercise exerted its effects via improvement of known cardiovascular risk factors. While there was a tendency toward a lower HR for CHD in association with an increase in LTPA, it was not statistically significant. In contrast, significant reductions in risks for stroke and total mortality were seen, and HRs for both stroke and total mortality in the top tertile were approximately half of those in the bottom tertile. The significance in total mortality was not affected by adjustment for lifestyle factors, clinical variables and medications (Table 2). Although stroke risk in the top tertile was significant after adjustment for age, sex and diabetes duration, HRs for the top tertile became of borderline significance (but still with a significant $p$ value for trend, 0.0495) after adjustment for lifestyle factors; the $p$ value was more than 0.1 after additional adjustment for clinical variables. On the other hand, analysis by a competing risk model that aimed to exclude the influence of mortality cases when assessing associations between LTPA and CHD or stroke did not fundamentally change the results (Table 2).

Probability in each outcome according to the tertile of LTPA determined by Kaplan-Meier analysis is shown in Fig. 1. A consistently lower risk of stroke and total mortality was found in the top tertile. Subgroup analysis (Fig. 2) revealed that among men or non-smokers the HR for stroke in individuals in the top tertile of LTPA was significantly lower than in those in the bottom tertile. Similarly, among those who were 60 years or older, were non-smokers, had a sedentary occupation, were not obese $\left(\mathrm{BMI}<25 \mathrm{~kg} / \mathrm{m}^{2}\right)$, had hypertension (systolic blood pressure $\geq 130 \mathrm{mmHg}$, diastolic blood pressure $\geq 80 \mathrm{mmHg}$ or those taking medication for hypertension) or who did not have dyslipidaemia (LDLcholesterol $<3.10 \mathrm{mmol} / 1[120 \mathrm{mg} / \mathrm{dl}]$, triacylglycerol $<1.69 \mathrm{mmol} / 1[150 \mathrm{mg} / \mathrm{dl}]$, HDL-cholesterol $\geq 1.03 \mathrm{mmol} /$ $1[40 \mathrm{mg} / \mathrm{dl}]$ and those not taking medication for dyslipidaemia), the HR for total mortality was lower in the top tertile than in the bottom tertile of LTPA. However, there were no significant interactions among these factors, suggesting lack of clear evidence of heterogeneity regarding the effect of LTPA on outcomes (Fig. 2).

\section{Discussion}

In comparison with type 2 diabetic patients in Western countries, those in East Asian countries, including Japan, are known to have quite different features regarding cardiovascular complications and their risk factors [22, 28-30]. Diabetic individuals in East Asian countries have a much lower degree of obesity and a much lower incidence of CHD 
Table 1 Baseline characteristics of the 1,702 Japanese patients with type 2 diabetes according to tertile of LTPA

\begin{tabular}{|c|c|c|c|c|c|}
\hline $\begin{array}{l}\text { Characteristic } \\
\text { No. of patients }\end{array}$ & $\begin{array}{l}\text { Total } \\
n=1702\end{array}$ & $\begin{array}{l}\text { Tertile } 1(\leq 3.7 \\
\text { MET h/week }) \\
n=551\end{array}$ & $\begin{array}{l}\text { Tertile } 2(3.8-15.3 \\
\text { MET h/week) } \\
n=589\end{array}$ & $\begin{array}{l}\text { Tertile } 3 \text { ( } \geq 15.4 \\
\text { MET h/week) } \\
n=562\end{array}$ & $p$ for trend \\
\hline LTPA (MET h/week) & $15.5 \pm 20.8$ & $0.8 \pm 1.1$ & $9.1 \pm 3.8$ & $36.8 \pm 24.4$ & $<0.01$ \\
\hline Women $(\%)$ & 47.1 & 48.3 & 48.4 & 44.5 & 0.20 \\
\hline Age (years) & $58.5 \pm 6.9$ & $57.9 \pm 7.2$ & $58.7 \pm 6.8$ & $59.0 \pm 6.7$ & 0.01 \\
\hline Diabetes duration (years) & $11.0 \pm 7.1$ & $10.4 \pm 6.6$ & $11.0 \pm 7.3$ & $11.6 \pm 7.4$ & 0.01 \\
\hline Sedentary occupation (\%) & 74.1 & 67.1 & 75.6 & 77.8 & $<0.01$ \\
\hline BMI $\left(\mathrm{kg} / \mathrm{m}^{2}\right)$ & $23.0 \pm 3.0$ & $23.2 \pm 3.2$ & $23.0 \pm 3.0$ & $22.7 \pm 2.9$ & 0.01 \\
\hline Waist circumference $(\mathrm{cm})$ & $79.4 \pm 9.2$ & $80.0 \pm 9.4$ & $79.6 \pm 9.1$ & $78.5 \pm 9.0$ & 0.01 \\
\hline Systolic blood pressure (mmHg) & $131.7 \pm 16.3$ & $131.5 \pm 16.4$ & $132.1 \pm 16.5$ & $131.6 \pm 16.1$ & 0.88 \\
\hline Diastolic blood pressure (mmHg) & $76.7 \pm 9.9$ & $76.8 \pm 10.0$ & $76.8 \pm 10.0$ & $76.6 \pm 9.9$ & 0.71 \\
\hline $\mathrm{HbA}_{1 \mathrm{c}}(\mathrm{JDS})(\%)$ & $7.9 \pm 1.3$ & $8.0 \pm 1.4$ & $7.8 \pm 1.2$ & $7.8 \pm 1.2$ & $<0.01$ \\
\hline $\mathrm{HbA}_{1 \mathrm{c}}(\mathrm{IFCC})(\mathrm{mmol} / \mathrm{mol})$ & $67.0 \pm 14.2$ & $68.8 \pm 15.6$ & $66.3 \pm 13.4$ & $66.0 \pm 13.4$ & $<0.01$ \\
\hline Fasting plasma glucose (mmol/l) & $8.85 \pm 2.41$ & $9.10 \pm 2.59$ & $8.71 \pm 2.18$ & $8.77 \pm 2.45$ & 0.03 \\
\hline LDL-cholesterol (mmol/1) & $3.17 \pm 0.83$ & $3.18 \pm 0.84$ & $3.17 \pm 0.85$ & $3.16 \pm 0.78$ & 0.67 \\
\hline HDL-cholesterol (mmol/l) & $1.42 \pm 0.44$ & $1.37 \pm 0.42$ & $1.41 \pm 0.42$ & $1.48 \pm 0.45$ & $<0.01$ \\
\hline Triacylglycerols $^{\mathrm{a}}(\mathrm{mmol} / \mathrm{l})$ & $1.15 \pm 0.80$ & $1.21 \pm 0.80$ & $1.18 \pm 0.84$ & $1.08 \pm 0.72$ & 0.05 \\
\hline Treated by insulin/OHA without insulin (\%) & $22.1 / 65.7$ & $22.9 / 68.6$ & $21.3 / 64.5$ & $22.1 / 64.2$ & $0.76 / 0.13$ \\
\hline Use of agents for hypertension (\%) & 25.9 & 30.7 & 24.6 & 22.4 & $<0.01$ \\
\hline Use of agents for dyslipidaemia (\%) & 24.0 & 24.9 & 23.6 & 23.4 & 0.55 \\
\hline Current smoker $(\%)$ & 27.6 & 31.0 & 30.2 & 22.1 & $<0.01$ \\
\hline Energy intake (kJ/day) & $7,183 \pm 1,469$ & $7,145 \pm 1,540$ & $7,217 \pm 1,473$ & $7,175 \pm 1,415$ & 0.82 \\
\hline Saturated fatty acid intake $(\mathrm{g})$ & $15.4 \pm 5.1$ & $15.4 \pm 5.4$ & $15.5 \pm 5.1$ & $15.2 \pm 4.9$ & 0.73 \\
\hline Dietary fibre intake $(\mathrm{g})$ & $14.6 \pm 5.2$ & $14.0 \pm 5.4$ & $14.7 \pm 5.2$ & $15.1 \pm 5.1$ & $<0.01$ \\
\hline $\begin{array}{l}\text { Ethanol intake (per day): never, } 3 \\
\text { drinks or less, more than } 3 \text { drinks }(\%)^{\text {b }}\end{array}$ & $62.1 / 31.5 / 6.4$ & $66.5 / 26.9 / 6.6$ & $61.9 / 31.7 / 6.3$ & $57.9 / 35.8 / 6.3$ & $<0.01 /<0.01 / 0.83$ \\
\hline \multicolumn{6}{|l|}{ No. of outcome incidents } \\
\hline CHD & 114 & 38 & 42 & 34 & - \\
\hline Stroke & 89 & 33 & 33 & 23 & - \\
\hline Mortality & 69 & 26 & 27 & 16 & - \\
\hline
\end{tabular}

Data are means ( \pm SD for continuous variables)

${ }^{a}$ Median (interquartile range)

b 'One drink' is equivalent to $12.6 \mathrm{~g}$ of ethanol based on the US Department of Agriculture definition

OHA, oral hypoglycaemic agent

than those in Western countries [28, 31]. Furthermore, cardiovascular disease is not necessarily a leading cause of death among diabetic patients in Japan [32], which is in distinct contrast to Western patients with diabetes [1]. Despite these differences, only two studies have prospectively investigated associations between physical activity and mortality and morbidity in Asian populations with diabetes $[4,7]$. This is notable, as Asian diabetic patients account for more than $60 \%$ of the world's diabetes population [28]. One was a recent report from Taiwan [7] that used a self-reported bivariate response of whether exercise was performed regularly as the only physical activity variable. The other study was from Japan [4] and involved only individuals with diabetes who were more than 65 years of age and demonstrated dose-dependent effects of physical activity that were evaluated and scored, although not by the use of universal MET $h$ units. Therefore, information on the recommended level of physical activity required to prevent complications in Asian patients with diabetes is scarce.

The current results for Japanese individuals with type 2 diabetes revealed that 15.4 MET h/week or more of LTPA was associated with a significant reduction in risk of stroke and total deaths (by approximately half) compared with 3.7 MET h/weekor less of LTPA. The cut-off of 15.4 MET h/ week in the current analysis corresponds to $2.2 \mathrm{MET} h /$ day which is, for example, equivalent to $30 \mathrm{~min} /$ day of brisk walking (3.5 miles [5.6 km]/h) and is 4.3 MET [23]. This is 
Table 2 HRs with 95\% CIs for LTPA according to tertiles (HRs for lowest tertile [ $\leq 3.7 \mathrm{MET} \mathrm{h} / \mathrm{week}$ ] as a reference) for cardiovascular disease (CHD and stroke) or total mortality risk analysed by Cox regression or competing risk regression

\begin{tabular}{|c|c|c|c|c|c|c|c|c|c|c|}
\hline \multirow[t]{3}{*}{ Analysis } & \multicolumn{5}{|l|}{ Cox regression } & \multicolumn{5}{|c|}{$\begin{array}{l}\text { Competing risk regression adjusted for non-cardiovascular } \\
\text { death }\end{array}$} \\
\hline & \multicolumn{2}{|c|}{$\begin{array}{l}\text { Tertile } 2 \text { (LTPA } \\
3.8-15.3 \mathrm{MET} \text { h/week) } \\
\text { (vs Tertile } 1[\text { LTPA } \\
\leq 3.7 \mathrm{MET} \mathrm{h/week])}\end{array}$} & \multicolumn{2}{|c|}{$\begin{array}{l}\text { Tertile } 3(\text { LTPA } \geq 15.4 \\
\text { MET h/week) }(v s \\
\text { Tertile } 1 \text { [LTPA } \\
\leq 3.7 \text { MET h/week]) }\end{array}$} & \multirow[t]{2}{*}{$p$ for trend } & \multicolumn{2}{|c|}{$\begin{array}{l}\text { Tertile } 2 \text { (LTPA } \\
3.8-15.3 \text { MET } \\
\text { h/week) (vs Tertile } \\
1 \text { [LTPA } \leq 3.7 \text { MET } \\
\text { h/week]) }\end{array}$} & \multicolumn{2}{|c|}{$\begin{array}{l}\text { Tertile } 3 \text { (LTPA } \geq 15.4 \\
\text { MET h/week) (vs Tertile } \\
1[\text { LTPA } \leq 3.7 \text { MET } \\
\text { h/week]) }\end{array}$} & \multirow[t]{2}{*}{$p$ for trend } \\
\hline & $\operatorname{HR}(95 \% \mathrm{CI})$ & $p$ & HR $(95 \%$ CI) & $p$ & & HR $(95 \%$ CI $)$ & $p$ & HR $(95 \% \mathrm{CI})$ & $p$ & \\
\hline \multicolumn{11}{|l|}{ CHD } \\
\hline Model $1^{\mathrm{a}}$ & $0.98(0.63,1.52)$ & 0.92 & $0.77(0.48,1.25)$ & 0.29 & 0.29 & $0.98(0.63,1.53)$ & 0.92 & $0.80(0.50,1.29)$ & 0.36 & 0.35 \\
\hline Model $2^{\mathrm{b}}$ & $0.97(0.57,1.65)$ & 0.91 & $0.71(0.40,1.24)$ & 0.23 & 0.19 & $0.95(0.55,1.67)$ & 0.87 & $0.73(0.41,1.32)$ & 0.30 & 0.26 \\
\hline Model $3^{\mathrm{c}}$ & $1.00(0.58,1.71)$ & 0.99 & $0.77(0.43,1.37)$ & 0.37 & 0.34 & $0.99(0.57,1.75)$ & 0.98 & $0.78(0.42,1.43)$ & 0.42 & 0.37 \\
\hline Model $4^{\mathrm{d}}$ & $0.96(0.56,1.65)$ & 0.87 & $0.77(0.43,1.38)$ & 0.38 & 0.35 & $0.96(0.53,1.71)$ & 0.88 & $0.78(0.42,1.43)$ & 0.42 & 0.39 \\
\hline \multicolumn{11}{|l|}{ Stroke } \\
\hline Model 1 & $0.78(0.48,1.27)$ & 0.32 & $0.55(0.32,0.94)$ & 0.03 & 0.03 & $0.78(0.48,1.28)$ & 0.33 & $0.55(0.33,0.94)$ & 0.03 & 0.03 \\
\hline Model 2 & $1.08(0.58,2.01)$ & 0.80 & $0.49(0.23,1.03)$ & 0.06 & 0.0495 & $1.09(0.58,2.03)$ & 0.79 & $0.49(0.23,1.07)$ & 0.07 & 0.04 \\
\hline Model 3 & $1.23(0.65,2.34)$ & 0.53 & $0.56(0.26,1.20)$ & 0.13 & 0.10 & $1.24(0.65,2.36)$ & 0.51 & $0.56(0.25,1.26)$ & 0.16 & 0.10 \\
\hline Model 4 & $1.27(0.67,2.43)$ & 0.47 & $0.57(0.26,1.23)$ & 0.15 & 0.12 & $1.28(0.67,2.44)$ & 0.46 & $0.57(0.25,1.30)$ & 0.18 & 0.12 \\
\hline \multicolumn{11}{|c|}{ CHD or stroke } \\
\hline Model 1 & $0.89(0.61,1.28)$ & 0.52 & $0.71(0.48,1.05)$ & 0.09 & 0.09 & $0.89(0.61,1.28)$ & 0.52 & $0.73(0.50,1.08)$ & 0.11 & 0.11 \\
\hline Model 2 & $0.94(0.60,1.46)$ & 0.77 & $0.61(0.38,0.99)$ & 0.04 & 0.03 & $0.93(0.59,1.46)$ & 0.75 & $0.64(0.39,1.04)$ & 0.07 & 0.052 \\
\hline Model 3 & $0.96(0.62,1.50)$ & 0.86 & $0.67(0.41,1.09)$ & 0.10 & 0.09 & $0.96(0.61,1.51)$ & 0.86 & $0.67(0.41,1.11)$ & 0.12 & 0.09 \\
\hline Model 4 & $0.96(0.61,1.50)$ & 0.85 & $0.68(0.42,1.11)$ & 0.12 & 0.10 & $0.96(0.61,1.51)$ & 0.85 & $0.68(0.41,1.13)$ & 0.14 & 0.11 \\
\hline \multicolumn{11}{|c|}{ Total mortality } \\
\hline Model 1 & $0.80(0.46,1.38)$ & 0.42 & $0.49(0.26,0.91)$ & 0.02 & 0.02 & - & - & - & - & - \\
\hline Model 2 & $0.80(0.43,1.49)$ & 0.49 & $0.41(0.20,0.87)$ & 0.02 & 0.02 & - & - & - & - & - \\
\hline Model 3 & $0.85(0.46,1.59)$ & 0.62 & $0.46(0.22,0.98)$ & 0.04 & 0.04 & & & & & \\
\hline Model 4 & $0.88(0.47,1.64)$ & 0.68 & $0.47(0.22,0.99)$ & 0.047 & 0.046 & - & - & - & - & - \\
\hline
\end{tabular}

${ }^{\text {a }}$ Model 1, adjusted by age, sex, diabetes duration

${ }^{\mathrm{b}}$ Model 2, model 1 plus lifestyle factors (smoking, energy/ethanol intake, dietary fibre, saturated fatty acid and type of occupation)

${ }^{\mathrm{c}}$ Model 3, model 2 plus clinical variables (BMI, $\mathrm{HbA}_{1 \mathrm{c}}$, systolic blood pressure, LDL-cholesterol, HDL-cholesterol, triacylglycerols)

${ }^{\mathrm{d}}$ Model 4, model 3 plus treatment with insulin, oral hypoglycaemic agents, antihypertensive agents or lipid-lowering agents

also relatively close to, but somewhat more than, the amount recommended by the Joint Position Statement of the American Diabetes Association/American College of Sports Medicine [2] and the Scientific Statement of the American Heart Association [3], both of which recommend $150 \mathrm{~min} /$ week of exercise of moderate or greater intensity. According to previous studies using almost identical quantitative methods for men [18] and women [21] in the USA, significantly reduced multivariate-adjusted risk (approximate HR 0.6) for total cardiovascular disease or total mortality was observed from approximately 12-16 MET $\mathrm{h} /$ week, which is close to our cut-off.

A characteristic finding of the current analysis was that among cardiovascular events stroke and not CHD was significantly reduced by LTPA, a result different from that found in diabetic women in the USA [21]. Interestingly, however, even in those results [21], a significantly reduced incidence of ischaemic stroke was seen beginning with fewer mean hours of moderate-to-vigorous activity per week (i.e. 2-3.9 h/week) compared with the significant reduction in CHD that was observed starting with 4-6.9 h/week. This suggests that exercise could be more effective in preventing stroke than CHD. Significantly decreased mortality risks from cerebrovascular or non-CHD cardiovascular disease, but not heart disease or CHD, by exercise were also reported in other individuals with diabetes in the USA [19] as well as in Japanese women in the general population [33]. While this manuscript was in preparation, analysis of another Japanese diabetic cohort that included only individuals over the age of 65 years demonstrated that physical activity as 
a

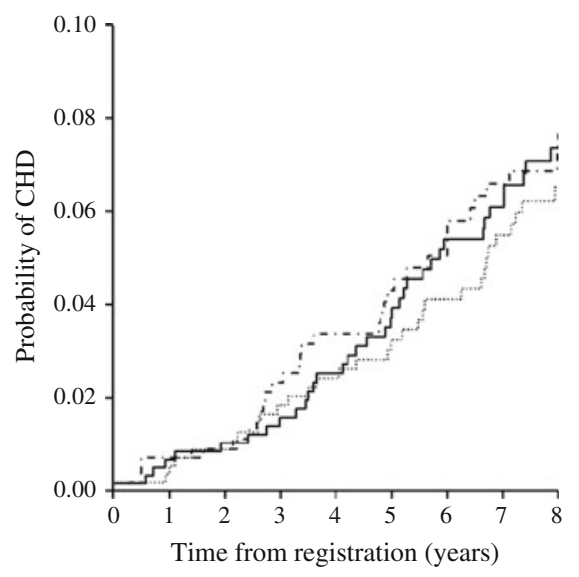

No. at risk

T1 of LTPA $\begin{array}{lllllllll}551 & 542 & 508 & 473 & 443 & 391 & 375 & 344 & 235\end{array}$ T2 of LTPA $\begin{array}{llllllllll}589 & 580 & 559 & 533 & 503 & 465 & 430 & 391 & 283\end{array}$

T3 of LTPA $562 \quad 556 \quad 535 \quad 511 \quad 491 \quad 455 \quad 427 \quad 403 \quad 289$

b

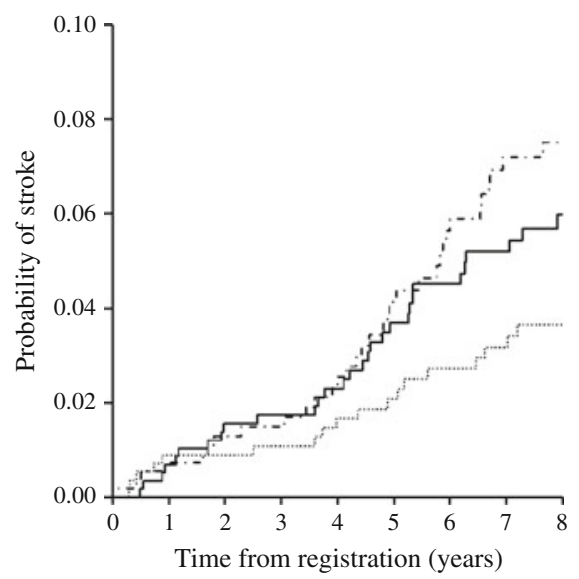

No. at risk

$\begin{array}{llllllllll}\text { T1 of LTPA } & 551 & 542 & 506 & 478 & 451 & 392 & 375 & 343 & 237\end{array}$

T2 of LTPA $\begin{array}{llllllllll}589 & 581 & 557 & 532 & 504 & 469 & 438 & 397 & 286\end{array}$

T3 of LTPA 562555536516497464435414301

\section{C}

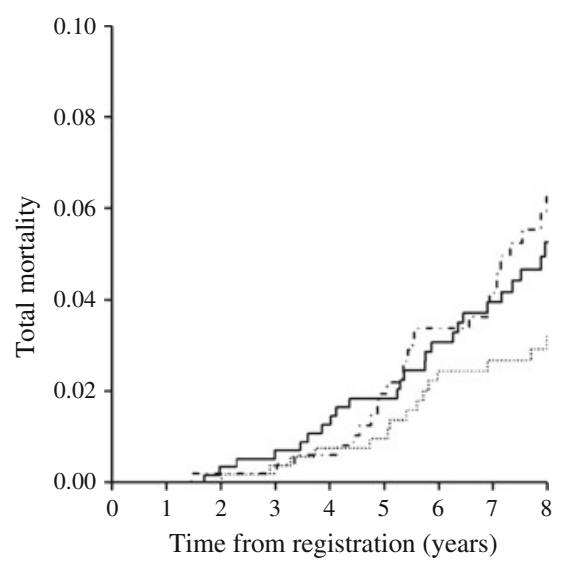

No. at risk

T1 of LTPA $551 \quad 546 \quad 515 \quad 486 \quad 464 \quad 410 \quad 391 \quad 367 \quad 248$

T2 of LTPA $589 \begin{array}{lllllllll}584 & 567 & 545 & 521 & 489 & 457 & 418 & 302\end{array}$

T3 of LTPA $562 \quad 558 \quad 542 \quad 523 \quad 504 \quad 478 \quad 448 \quad 427 \quad 312$
4Fig. 1 Probability of coronary heart disease (a), stroke (b) or total mortality (c) according to tertiles of LTPA determined by the KaplanMeier method. Broken line, tertile 1 (T1) of LTPA ( $\leq 3.7 \mathrm{MET} \mathrm{h/week);}$ solid line, tertile 2 (T2) of LTPA (3.8-15.3 MET h/week); dotted line, tertile 3 (T3) of LTPA $(\geq 15.4$ MET h/week)

evaluated and scored was significantly associated only with cerebrovascular events and not with cardiac events [4].

The precise mechanisms for these findings cannot be clarified merely from epidemiological studies. Although statistically nonsignificant, since a weak tendency for a decrease in HR for CHD across LTPA tertiles was observed in our cohort, it is possible that the relationship between CHD and LTPA would become significant with a longer period of observation. Because the biological mechanisms for stroke prevention by physical activity in patients with type 2 diabetes are only partially understood [34], the possibility exists that exercise ameliorates undetermined cardiovascular risk factors, such as quality of life [2] or other health behaviours [3], which more strongly affect stroke risk than CHD risk. This possibility should be investigated in the future.

The significant risk reduction in stroke by LTPA was weakened after stepwise adjustment for lifestyle factors and clinical variables, which suggested that some of the involved elements confounded the association although these adjustments did not compromise the beneficial effects of LTPA. However, these findings could be helpful in understanding the mechanisms behind the associations. Individual adjustments for each lifestyle factor instead of simultaneous adjustment for all lifestyle factors suggested that dietary factors (intake of energy, saturated fat and dietary fibre) had a relatively larger effect than the other lifestyle factors, suggesting that individuals who exercised more also had a tendency to pay more attention to the amount and content of meals (see ESM Table 1). On the other hand, individual adjustments for each clinical variable instead of simultaneous adjustment for all clinical variables suggested that triacylglycerol and LDL-cholesterol had a larger effect than the other clinical variables, indicating that LTPA might have exerted its effect on stroke reduction partly through ameliorating serum lipids (see ESM Table 1). Even though it became nonsignificant, the fully adjusted HR and its $p$ value for stroke did not alter dramatically from those when adjusted only by age, sex and diabetes duration. This suggests that undetermined risk factors associated with physical activity should exist. This is also supported in part by the results of subgroup analysis of the risk of stroke and total mortality, which indicated that greater LTPA was not necessarily associated with lower risk in those who had typical cardiovascular risk factors.

The current results suggested that the effect of exercise on total mortality was independent of lifestyle factors and clinical variables, which included typical cardiovascular risk 
Fig. 2 Subgroup analysis adjusted for age, sex, duration of diabetes and lifestyle factors. HRs for CHD (a), stroke (b) and total mortality (c) are shown for tertile 3 vs tertile 1 (reference) for LTPA. Bars in the figure indicate $95 \%$ CIs. Normal weight is defined as BMI $<25 \mathrm{~kg} / \mathrm{m}^{2}$; normotension is defined as systolic blood pressure $<130 \mathrm{mmHg}$, diastolic blood pressure $<80 \mathrm{mmHg}$ and not under medication for hypertension; and normolipidaemia is defined as serum levels of LDLcholesterol $<3.10 \mathrm{mmol} / 1(120 \mathrm{mg} / \mathrm{dl})$, triacylglycerols $<1.69 \mathrm{mmol} / \mathrm{l}$ $(150 \mathrm{mg} / \mathrm{dl})$, HDL-cholesterol $>1.03 \mathrm{mmol} / \mathrm{l}(40 \mathrm{mg} / \mathrm{dl})$ and not under medication for dyslipidaemia. To convert values for $\mathrm{HbA}_{1 \mathrm{c}}$ in \% into $\mathrm{mmol} / \mathrm{mol}$, subtract 2.15 and multiply by 10.929

factors such as smoking, dyslipidaemia and hypertension. Since it is well known that the majority of Western individuals with diabetes die from cardiovascular diseases and that exercise is known to improve cardiovascular risk factors [1], it is natural to consider that physical activity ameliorates mortality mainly through preventing cardiovascular events $[2,3]$. In this study, we unfortunately could not evaluate the effect of CHD and stroke on total mortality since a causespecific mortality could not be calculated because of the limited number of events. Although difficult to prove, the current findings suggest that the significant reduction in mortality related to LTPA was not derived from a reduction in CHD and stroke but from a reduction in causes of death other than CHD or stroke. Cancer was the top cause of death among our study participants, which is in accordance with previously reported results in the diabetic [32] as well as the general [33] population in Japan. This might suggest that an enhanced risk of cancer by diabetes [35] could be somewhat counterbalanced by the effect of exercise. There is strong and consistent evidence that physical activity reduces the risk of cancer $[33,35,36]$, including that in the general Japanese population [33]. The amount of physical activity recommended for cancer prevention (i.e. 30-60 min of moderate- or vigorous-intensity exercise at least 5 days per week) [36] is close to that recommended for diabetic persons $[2,3]$.

Our study has several strengths. It was a large-scale study with nationwide sampling from nearly 60 institutes, LTPA was quantified using a universal MET score, and dietary data were available. The results were also confirmed by competing risk models as well as detailed adjustment models to clarify the underlying mechanisms. Nevertheless, some limitations of this investigation deserve consideration. One is the observational design, which could allow the possibility of unmeasured confounders. The limited number of participants prevented us from analysing data separately by sex or by quartiles or quintiles instead of tertiles. The necessity of a large-scale cohort study in the future is implied by our results. All of our participants were recruited from clinics of universities or large general hospitals, which might limit the extrapolation of the results to primary care settings. However, they were not necessarily tertiary-care a

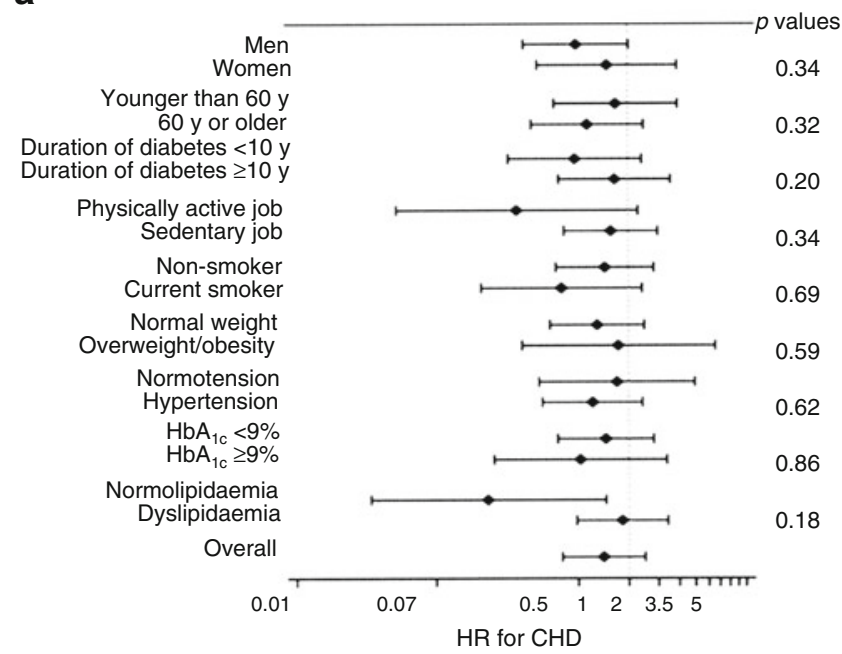

b

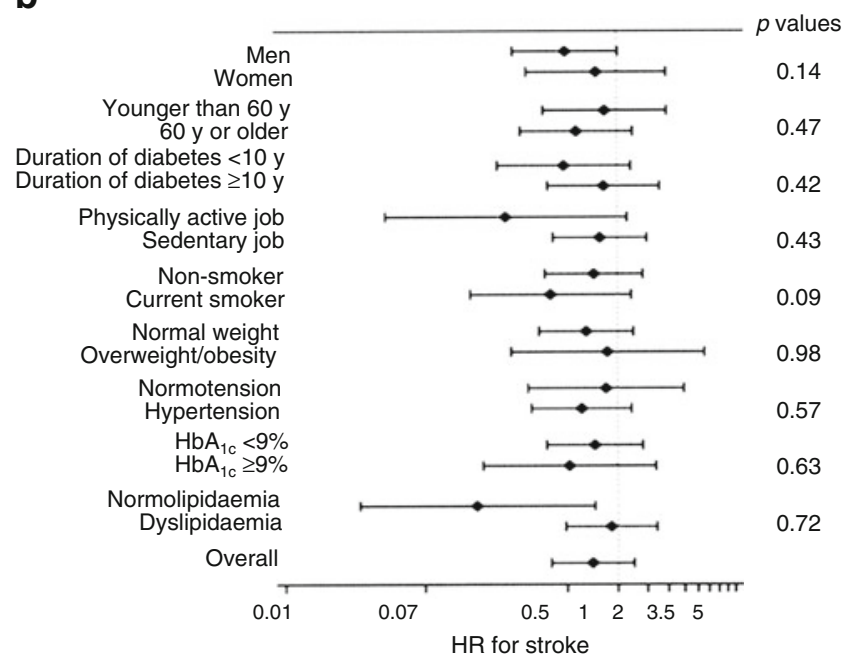

C

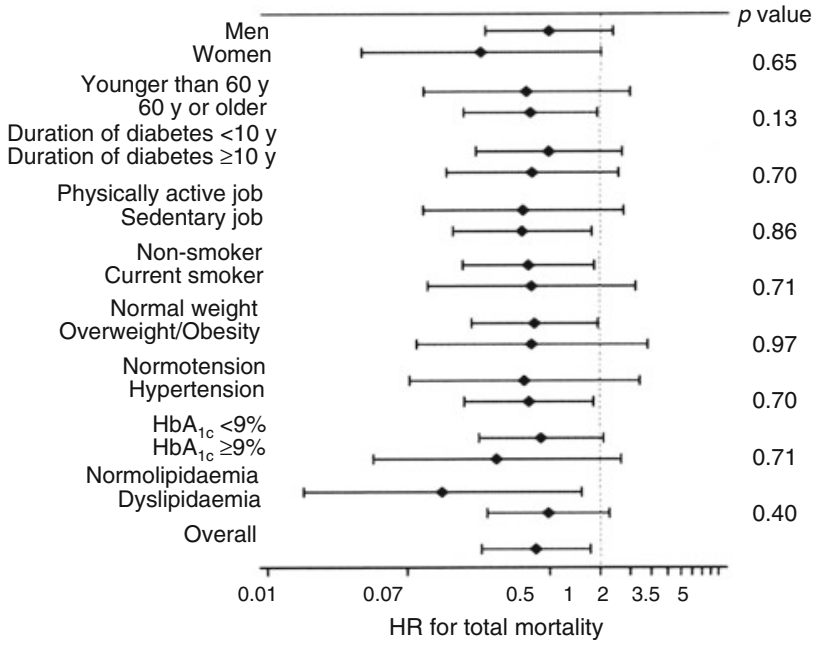

patients since the health insurance system in Japan allows patients to freely choose outpatient clinics regardless of severity or degree of progression of diabetes. We could not 
determine physical activity related to occupation and commuting since we did not survey working hours per week or commuting methods. However, $\mathrm{Hu}$ and colleagues [37] reported that physical activity related to occupation and commuting significantly affected the results in their cohort of individuals with diabetes [10], although adjustment by individual or dichotomous (physically active/ sedentary) classifications of occupations did not affect our results. It might be meaningful to show the effects of LTPA independently of occupation since an occupation per se is not an easily 'modifiable' element for many individuals. Only baseline data, including those using medication, were considered in this analysis; however, adjustment by baseline use of medication did not fundamentally change our results, therapeutic management during the follow-up period could have influenced these results. In fact, we found a substantial increase in usage of antihypertensive and hypolipidaemic agents during the follow-up period, as previously reported [38]. However, it would not be appropriate to adjust for the influence of medications during follow-up in our analysis, as these variables can be outcomes of low LTPA at baseline. We could only show that clinical variables according to tertiles of LTPA remained quite stable during the observational period (see ESM Fig. 1).

Loss to follow-up is an inevitable problem in most cohort studies. In this study, the 8-year follow-up rate was not necessarily high, and LTPA, total energy intake and treatment with insulin were associated with follow-up status (see ESM Table 2). Theoretically, in such cases, a valid analysis requires inclusion of all observed prognostic factors associated with follow-up status into the model, even under the assumption of 'missing at random'. Taken together, HRs should be estimated with adjustment for LTPA, total energy intake and treatment by insulin, and Model 4 in Table 2 is the least likely to be biased according to this theoretical consideration. We did not assess cardiorespiratory fitness, which is known to be closely related to cardiovascular events in general [39] and in diabetic populations [40, 41], although the beneficial effects of LTPA are not fully explained only by cardiorespiratory fitness [42, 43].

In conclusion, in our cohort of Japanese individuals with type 2 diabetes, an LTPA level of 15.4 MET h/ week of more was associated with a significantly lower risk of stroke through, at least partially, ameliorating the effects of combinations of known cardiovascular risk factors. Higher LTPA was also associated with significantly reduced total mortality but independent of cardiovascular risk factors or events. These findings, which imply differences from Western diabetic populations, should be considered in the clinical management of East Asians with diabetes.
Acknowledgements We thank the patients, staff and diabetologists throughout Japan for their long-standing collaboration in this study. We are also indebted to the late R. Abe (Ohta-Nishinouchi Hospital) for his contribution in organising the physical activity project of the JDCS. Thanks are extended to S. Fukuya and Y. Maruyama (University of Tsukuba) for their excellent secretarial assistance.

Funding This study is supported by grants from the Ministry of Health, Labor and Welfare. The sponsor had no role in the design and conduct of the study.

Duality of interest The authors declare that there is no duality of interest associated with this manuscript.

Contribution statement All authors contributed to the conception and design of the study, acquisition, analysis and interpretation of data and drafting and editing the manuscript. All of the authors approved the final version of the manuscript. H. Sone had full access to all of the data in the study and takes responsibility for the integrity of the data and the accuracy of the data analysis.

\section{References}

1. Chudyk A, Petrella RJ (2011) Effects of exercise on cardiovascular risk factors in type 2 diabetes: a meta-analysis. Diabetes Care 34:1228-1237

2. Colberg SR, Sigal RJ, Fernhall B et al (2010) Exercise and type 2 diabetes: the American College of Sports Medicine and the American Diabetes Association: joint position statement executive summary. Diabetes Care 33:2692-2696

3. Marwick TH, Hordern MD, Miller T et al (2009) Exercise training for type 2 diabetes mellitus: impact on cardiovascular risk: a scientific statement from the American Heart Association. Circulation 119:3244-3262

4. Iijima K, Iimuro S, Shinozaki T et al (2012) Lower physical activity is a strong predictor of cardiovascular events in elderly patients with type 2 diabetes mellitus beyond traditional risk factors: the Japanese Elderly Diabetes Intervention Trial. Geriatr Gerontol Int 12(supp11):77-87

5. Cipollini F, Gussoni G, Pacifici R et al (2011) The influence of physical activity performed at $20-40$ years of age on cardiovascular outcomes in medical patients aged 65-75. Italian J Med 5:114-119

6. Nothlings U, Ford ES, Kroger J, Boeing H (2010) Lifestyle factors and mortality among adults with diabetes: findings from the European Prospective Investigation into Cancer and NutritionPotsdam study. J Diabetes 2:112-117

7. Lin CC, Li CI, Liu CS et al (2012) Impact of lifestyle-related factors on all-cause and cause-specific mortality in patients with type 2 diabetes: the Taichung Diabetes Study. Diabetes Care 35:105-112

8. Vepsalainen T, Soinio M, Marniemi J et al (2011) Physical activity, high-sensitivity C-reactive protein, and total and cardiovascular disease mortality in type 2 diabetes. Diabetes Care 34:1492-1496

9. de Fine Olivarius N, Siersma V, Nielsen AB, Hansen LJ, Rosenvinge L, Mogensen CE (2010) Predictors of mortality of patients newly diagnosed with clinical type 2 diabetes: a 5-year follow up study. BMC Endocr Disord 10:14

10. Hu G, Jousilahti P, Barengo NC, Qiao Q, Lakka TA, Tuomilehto J (2005) Physical activity, cardiovascular risk factors, and mortality among Finnish adults with diabetes. Diabetes Care 28:799-805

11. Gregg EW, Gerzoff RB, Caspersen CJ, Williamson DF, Narayan KM (2003) Relationship of walking to mortality among US adults with diabetes. Arch Intern Med 163:1440-1447 
12. Batty GD, Shipley MJ, Marmot M, Smith GD (2002) Physical activity and cause-specific mortality in men with type 2 diabetes/ impaired glucose tolerance: evidence from the Whitehall study. Diabet Med 19:580-588

13. Ford ES, DeStefano F (1991) Risk factors for mortality from all causes and from coronary heart disease among persons with diabetes. Findings from the National Health and Nutrition Examination Survey I Epidemiologic Follow-up Study. Am J Epidemiol 133:1220-1230

14. Nelson KM, Boyko EJ, Koepsell T (2010) All-cause mortality risk among a national sample of individuals with diabetes. Diabetes Care 33:2360-2364

15. Trichopoulou A, Psaltopoulou T, Orfanos P, Trichopoulos D (2006) Diet and physical activity in relation to overall mortality amongst adult diabetics in a general population cohort. J Intern Med 259:583-591

16. Wei M, Gibbons LW, Kampert JB, Nichaman MZ, Blair SN (2000) Low cardiorespiratory fitness and physical inactivity as predictors of mortality in men with type 2 diabetes. Ann Intern Med 132:605611

17. Moy CS, Songer TJ, LaPorte RE et al (1993) Insulin-dependent diabetes mellitus, physical activity, and death. Am J Epidemiol 137:74-81

18. Tanasescu M, Leitzmann MF, Rimm EB, Hu FB (2003) Physical activity in relation to cardiovascular disease and total mortality among men with type 2 diabetes. Circulation 107:2435-2439

19. Smith TC, Wingard DL, Smith B, Kritz-Silverstein D, BarrettConnor E (2007) Walking decreased risk of cardiovascular disease mortality in older adults with diabetes. J Clin Epidemiol 60:309317

20. Spencer EA, Pirie KL, Stevens RJ et al (2008) Diabetes and modifiable risk factors for cardiovascular disease: the prospective Million Women Study. Eur J Epidemiol 23:793-799

21. Hu FB, Stampfer MJ, Solomon C et al (2001) Physical activity and risk for cardiovascular events in diabetic women. Ann Intern Med 134:96-105

22. Sone H, Tanaka S, Tanaka S et al (2012) Comparison of various lipid variables as predictors of coronary heart disease in Japanese men and women with type 2 diabetes: subanalysis of the Japan Diabetes Complications Study (JDCS). Diabetes Care 35:11501157

23. Ainsworth BE, Haskell WL, Herrmann SD et al (2011) 2011 Compendium of Physical Activities: a second update of codes and MET values. Med Sci Sports Exerc 43:1575-1581

24. Takahashi K, Yoshimura Y, Kaimoto T, Kunii D, Komatsu T, Yamamoto S (2001) Validation of a food frequency questionnaire based on food groups for estimationg individual nutrient intake. Jpn J Nutr 59:221-232 [article in Japanese]

25. Saito K, Sone H, Kawai K et al (2007) Risk imparted by various parameters of smoking in Japanese men with type 2 diabetes on their development of microalbuminuria: analysis from the Tsukuba Kawai Diabetes Registry. Diabetes Care 30:1286-1288

26. The Statistics Bureau and the Director-General for Policy Planning of Japan MoIAaC (1997) Japan Standard Classification of Occupations. http://www.stat.go.jp/index/seido/shokgyou/. Accessed 10 December 2012 [in Japanese]
27. Schoenaker DA, Toeller M, Chaturvedi N, Fuller JH, SoedamahMuthu SS (2012) Dietary saturated fat and fibre and risk of cardiovascular disease and all-cause mortality among type 1 diabetic patients: the EURODIAB Prospective Complications Study. Diabetologia 55:2132-2141

28. Chan JC, Malik V, Jia W et al (2009) Diabetes in Asia: epidemiology, risk factors, and pathophysiology. JAMA 301:2129-2140

29. Sone H, Yamada N, Mizuno S, Aida R, Ohashi Y (2004) Alcohol use and diabetes mellitus. Ann Intern Med 141:408-409

30. Sone H, Tanaka S, Iimuro S et al (2011) Serum level of triglycerides is a potent risk factor comparable to LDL cholesterol for coronary heart disease in Japanese patients with type 2 diabetes: subanalysis of the Japan Diabetes Complications Study (JDCS). J Clin Endocrinol Metab 96:3448-3456

31. Sone H, Ito H, Ohashi Y, Akanuma Y, Yamada N (2003) Obesity and type 2 diabetes in Japanese patients. Lancet 361:85

32. Hotta N, Nakamura J, Iwamoto Y et al (2010) Causes of death in Japanese diabetics: a questionnaire survey of 18,385 diabetics over a 10-year period. J Diabet Invest 1:66-76

33. Inoue M, Iso H, Yamamoto $\mathrm{S}$ et al (2008) Daily total physical activity level and premature death in men and women: results from a large-scale population-based cohort study in Japan (JPHC study). Ann Epidemiol 18:522-530

34. Agosti V, Graziano S, Artiaco L, Sorrentino G (2009) Biological mechanisms of stroke prevention by physical activity in type 2 diabetes. Acta Neurol Scand 119:213-223

35. Giovannucci E, Harlan DM, Archer MC et al (2010) Diabetes and cancer: a consensus report. Diabetes Care 33:1674-1685

36. Friedenreich CM, Neilson HK, Lynch BM (2010) State of the epidemiological evidence on physical activity and cancer prevention. Eur J Cancer 46:2593-2604

37. Hu G, Eriksson J, Barengo NC et al (2004) Occupational, commuting, and leisure-time physical activity in relation to total and cardiovascular mortality among Finnish subjects with type 2 diabetes. Circulation 110:666-673

38. Sone H, Tanaka S, Iimuro S et al (2010) Long-term lifestyle intervention lowers the incidence of stroke in Japanese patients with type 2 diabetes: a nationwide multicentre randomised controlled trial (the Japan Diabetes Complications Study). Diabetologia 53:419-428

39. Kodama S, Saito K, Tanaka S et al (2009) Cardiorespiratory fitness as a quantitative predictor of all-cause mortality and cardiovascular events in healthy men and women: a meta-analysis. JAMA 301:2024-2035

40. Kokkinos P, Myers J, Nylen E et al (2009) Exercise capacity and all-cause mortality in African American and Caucasian men with type 2 diabetes. Diabetes Care 32:623-628

41. McAuley PA, Myers JN, Abella JP, Tan SY, Froelicher VF (2007) Exercise capacity and body mass as predictors of mortality among male veterans with type 2 diabetes. Diabetes Care 30:1539-1543

42. Tager IB, Hollenberg M, Satariano WA (1998) Association between self-reported leisure-time physical activity and measures of cardiorespiratory fitness in an elderly population. Am J Epidemiol 147:921-931

43. Lakka TA, Venalainen JM, Rauramaa R, Salonen R, Tuomilehto J, Salonen JT (1994) Relation of leisure-time physical activity and cardiorespiratory fitness to the risk of acute myocardial infarction. N Engl J Med 330:1549-1554 